
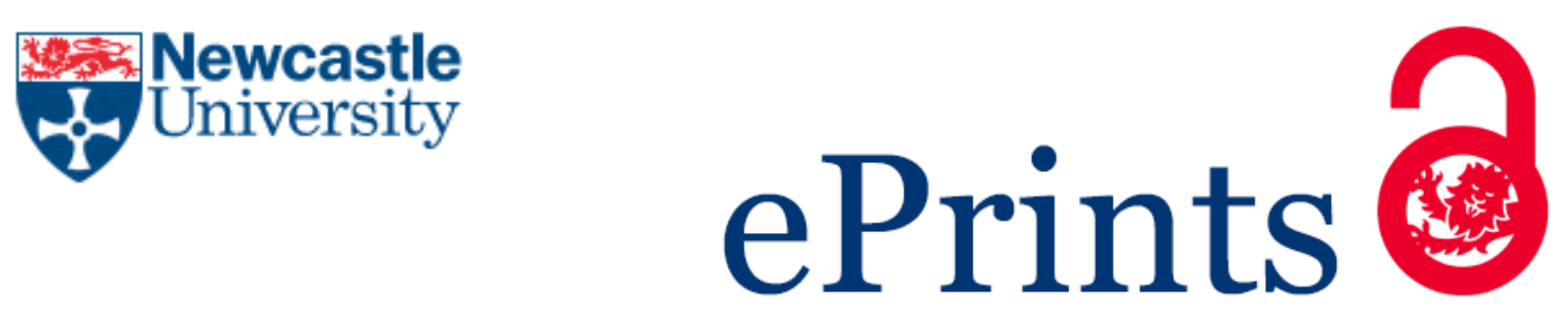

Nasirahmadi A, Hensel O, Edwards S, Sturm B. Automatic detection of mounting behaviours among pigs using image analysis. Computers and Electronics in Agriculture 2016, 124, 295-302.

\title{
Copyright:
}

(C) 2016. This manuscript version is made available under the CC-BY-NC-ND 4.0 license

DOI link to article:

http://dx.doi.org/10.1016/i.compag.2016.04.022

Date deposited:

$05 / 07 / 2016$

Embargo release date:

30 April 2017

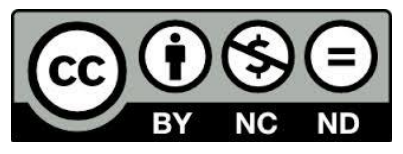

This work is licensed under a

Creative Commons Attribution-NonCommercial-NoDerivatives 4.0 International licence 


\section{Automatic detection of mounting behaviours among pigs using image analysis}

2

\section{Abozar Nasirahmadi ${ }^{1,2}$, Oliver Hensel ${ }^{2}$, Sandra Edwards ${ }^{1}$, Barbara Sturm ${ }^{1,2}$}

${ }^{1}$ School of Agriculture, Food and Rural Development, Newcastle University, Newcastle upon Tyne NE1 7RU, UK

${ }^{2}$ Department of Agricultural and Biosystems Engineering, University of Kassel, 37213 Witzenhausen, Germany

\section{Abstract}

Excessive mounting behaviours amongst pigs cause a high risk of poor welfare, arising from skin lesions, lameness and stress, and economic losses from reduced performance. The aim of this study was to develop a method for automatic detection of mounting events amongst pigs under commercial farm conditions by means of image processing. Two pens were selected for the study and were monitored for 20 days by means of top view cameras. The recorded video was then visually analysed for selecting mounting behaviours, and extracted images from the video files were subsequently used for image processing. An ellipse fitting technique was applied to localize pigs in the image. The intersection points between the major and minor axis of each fitted ellipse and the ellipse shape were used for defining the head, tail and sides of each pig. The Euclidean distances between head and tail, head and sides, the major and minor axis length of the fitted ellipse during the mounting were utilized for development of an algorithm to automatically identify a mounting event. The proposed method could detect mounting events with high level of sensitivity, specificity and accuracy, 94.5, 88.6 and $92.7 \%$, respectively. The results show that it is possible to use machine vision techniques in order to automatically detect mounting behaviours among pigs under commercial farm conditions.

\footnotetext{
${ }^{1}$ Corresponding author: abozar.nasirahmadi@ncl.ac.uk, a.nasirahmadi@gmail.com
} 
Keywords: Pig, Mounting behaviour, Image processing, Ellipse fitting.

\section{Introduction}

Mounting behaviours in pigs can be defined as when a pig lifts its two front legs and puts the two legs or its sternum on any part of the body or head of another pig; the mounted pig may stand or sit down during the mounting or move away to avoid being mounted (Hintze et al., 2013). Both male and female pigs perform mounting behaviour, with different frequencies (Rydhmer et al., 2006; Hemsworth and Tilbrook, 2007), and the behaviour occurs more frequently in overcrowded conditions (Faucitano, 2001). Mounting behaviour amongst pigs can increase the risk of injuries, such as bruises and damage to the skin when pigs mount one another and scratch the back with the claws of the forelimbs (Faucitano, 2001; Harley et al., 2014), and lameness or leg fractures (Rydhmer et al., 2004). These injuries and the general unrest in the group can have considerable negative economic consequences (Rydhmer et al., 2006). Although the level of activity declines with increasing weight, mounting behaviour (Thomsen et al., 2012), and skin lesions and lameness (Teixeira and Boyle, 2014), happen during the entire growing period of pigs. Investigations of the mounting behaviour of pigs have already been made in different studies. However, these have generally been carried out using direct visual observations to sample behaviour under experimental conditions, reflected by a small number of pigs in the pen. Hintze et al. (2013) developed an ethogram of different types of mounting behaviours and their consequences. According to their classification, sexual mounts were longer than non-sexual mounts and were associated with more screaming, which is an indicator of stress and reduced welfare in pigs, by the mounted animal.

Image processing techniques have increasingly been applied to pig farm management in recent years and different studies have been carried out on the development of machine vision 
51 tools for pig production. By using a CCD camera the amount of pigs' water usage was estimated automatically with an accuracy of $92 \%$ based on their head distances to the drinking nipples in the images (Kashiha et al., 2013). Pig herds have been monitored using the optical flow method developed by Gronskyte et al. (2015) for obtaining undesirable events in the slaughterhouse with high overall sensitivity and specificity. Lu et al. (2016) proposed automatic weight estimation of pigs using image processing systems. In order to identify aggressive behaviours among pigs, motion history features have been applied (Viazzi et al., 2014) resulting in an overall high accuracy and sensitivity. Thermal comfort and lying patterns of groups of pigs have also been investigated with a high degree of accuracy by applying image processing techniques (Shao and Xin, 2008; Costa et al., 2014; Nasirahmadi et al., 2015). Recently some more state-of-art image capture methods have been applied in farms in order to improve animal welfare and monitor performance. A Vicon 3D optoelectronic motion analysis system and the Kinect motion sensor have been used for pig lameness detection (Stavrakakis et al., 2015) and the proposed method could distinguish the sound from lame pigs. For estimation the weight of pigs (Kongsro, 2014) and broilers (Mortensen et al., 2016) 3D Kinect cameras have been used. Furthermore, backfat thickness of Holstein-Friesian cows was estimated using a time-to-flight camera by Weber et al. (2014).

Every year approximately 100 million male piglets are castrated in the EU countries to control risk of boar taint and undesirable male behaviours. Surgical castration is a painful and stressful event (Prunier et al., 2006; Hintze et al., 2013), and its abolition is currently being proposed. If systems with entire male pigs are adopted in consequence, employing an automated machine vision method as a non-contact way for monitoring mounting behaviours in pig farms could help to inform farm managers about the number of mounting events and identify pens requiring intervention. It would also facilitate large scale research into methods 
to reduce this behavioural problem. A method using low cost CCTV cameras would be more economically acceptable for farm mangers than one requiring investment in expensive high resolution cameras. However, no studies have yet been done on the topic of automated detection of mounting and the feasibility of a low-cost system for this requires evaluation. Hence, the main object of this research was to develop an automatic method for detection of mounting behaviours among pigs under commercial pig farm conditions by means of machine vison techniques and development of image analysis algorithms.

\section{Material and methods}

\subsection{Animal and data collection}

The study was carried out at a commercial pig farm in the UK and started after placement of pigs in the pen at about $30 \mathrm{~kg}$ live weight. A 20 day period of data collection was used to generate sufficient occurrences of mounting behaviour. Each pen had a dimension of $6.75 \mathrm{~m}$ wide $\times 3.10 \mathrm{~m}$ long, with a fully slatted floor, and contained $22-23$ pigs of mixed gender (entire males or females). All pens were equipped with a liquid feeding trough and one drinking nipple. During the experiment lights were switched on and video recording of the pigs in two of the pens were made. Each research pen was equipped with a CCVT camera (Sony RF2938, EXview HAD CCD, Board lens 3.6 mm, 90², Gyeonggi-do, South Korea) which was located centrally at 4.5 meters above the ground and pointing directly downward to get a top view. Video images from the cameras were recorded simultaneously for $24 \mathrm{~h}$ during the day and night and stored in the hard disk of a PC using Geovision software (Geovision Inc. California, USA) with a frame rate of $30 \mathrm{fps}$, at a resolution of $640 \times 480$ pixels. After downloading the recorded data, the video files were directly observed and labelled in order to evaluate peak times of mounting activity (Hintze et al., 2013). A sufficient number of occurrences of the behaviour for testing the automated approach were 
101 obtained using five days of $24 \mathrm{~h}$ activity selected from the available sample. Two periods

102

103

104

105

106

107

108

109

110

111

112

113 were selected ( $2 \mathrm{~h}$ between 09:30 to 11:30 AM; 3 h between 14:30 to 17:30 PM) for each day and pen, during which the number of mounting events was increased compared to other periods. The selected video files were then used for extracting frames for further processing.

\subsection{Image processing}

In this study CCTV cameras were used, and distortions are common for the low-end lenses of such cameras (Geys and Gool, 2007). In order to remove barrel distortion in the images, camera calibration was carried out using the 'Camera Calibration Toolbox' of MATLAB ${ }^{\circledR}$ (the Mathworks Inc., Natick, MA, USA) and 25 extracted images of a pattern plane were taken in different orientations for each camera (Wang et al., 2007) and projected on the pen surface. The extracted image samples used for the mounting analysis were subjected to a four-step image processing (Fig. 1). 


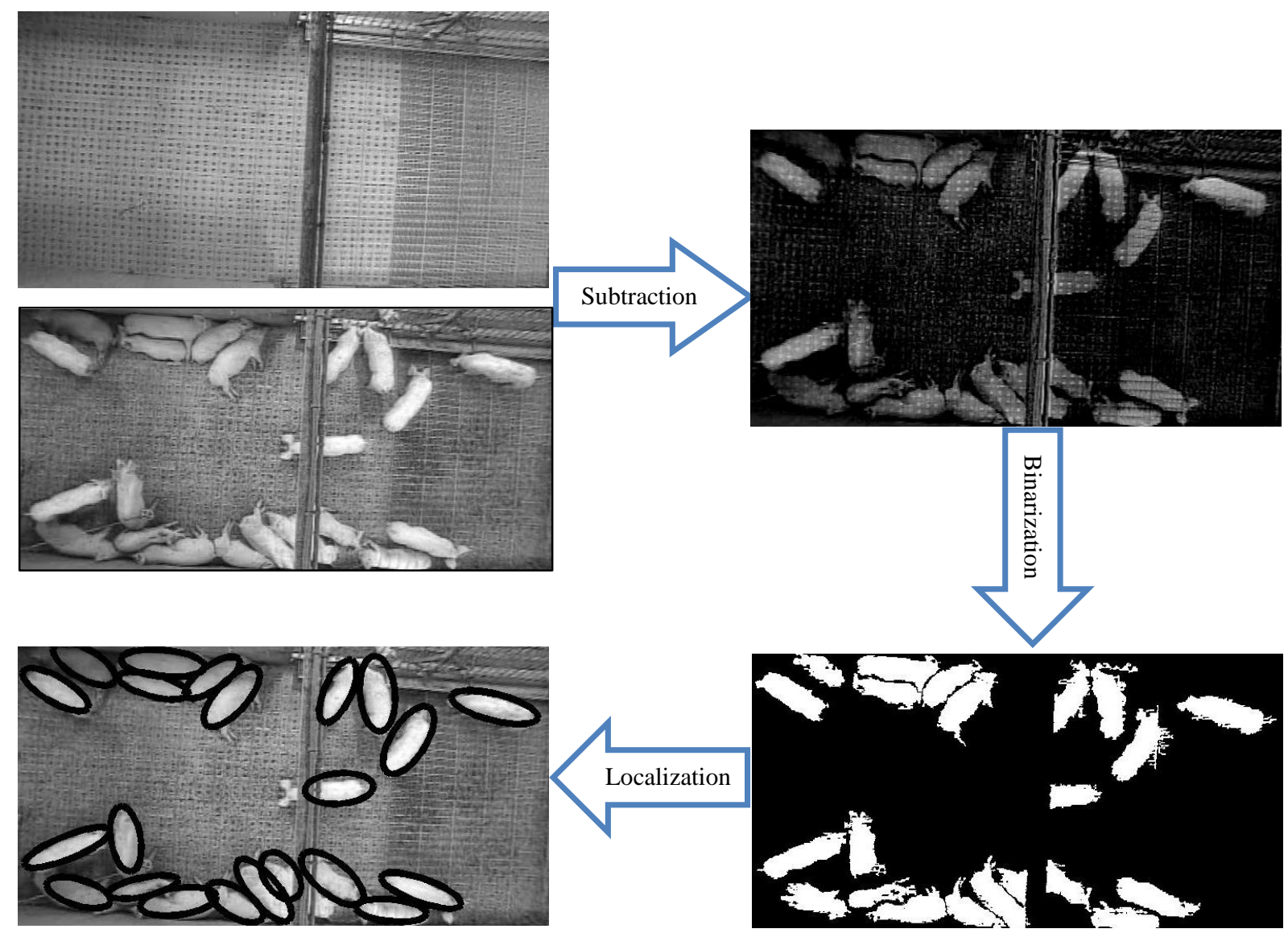

Fig.1. Image processing steps in this study; background (top left), grey image (middle left), subtracted image (top right), binary image (down right) and fitted ellipse (down left).

117 First step: in order to extract foreground objects (pigs) from the background (pen), a 118 background subtraction method was used.

119 Second step: a global threshold was applied using Otsu's method (Otsu, 1979) and the threshold was used to convert the greyscale image into a binary image.

121 Third step: disk structure of erosion and dilation for smoothing the edges was used, and then 122 small objects were removed from images by applying a morphological closing operator 123 (Gonzalez and Woods, 2007).

124 Forth step: to localize each pig body as an image, an ellipse fitting algorithm was applied 125 (O’Leary, 2004; Nasirahmadi et al., 2015) and ellipse parameters such as “major axis 
126 length", "minor axis length", “orientation" and "centroid" were calculated for all fitted

127 ellipses.

128

\section{2.3. Mounting behaviour detection}

130 The detection rule for pig mounting events in frame sequences is based on distance between 131 pigs, as normally a mounting pig gets close to another pig and then lifts its two front legs and 132 puts them on any part of the recipient or mounted pig (Fig. 2). The mounted pig may stand, 133 sit down or run away, and the duration of mounting can be short $(<1 \mathrm{~s})$, medium $(1-10 \mathrm{~s})$ or 134 long (>10-60s) (Hintze et al., 2013). Fig. 2 illustrates a video sequence for a mounting event 135 in a pen, where in frames (f1-f2) the distance between two pigs (mounting and mounted) 136 became less; this distance could be between the centre of two pigs or the head of one pig to 137 the tail of the next one. The mounting event happened in frames (f3-f5), in frame (f6) the 138 mounting/mounted pig moved away and the event finished. 


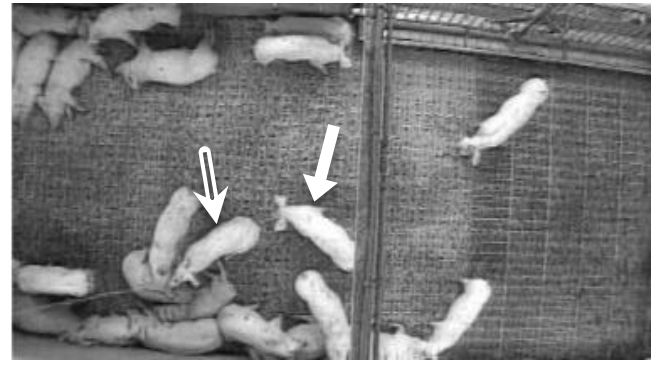

(f1)

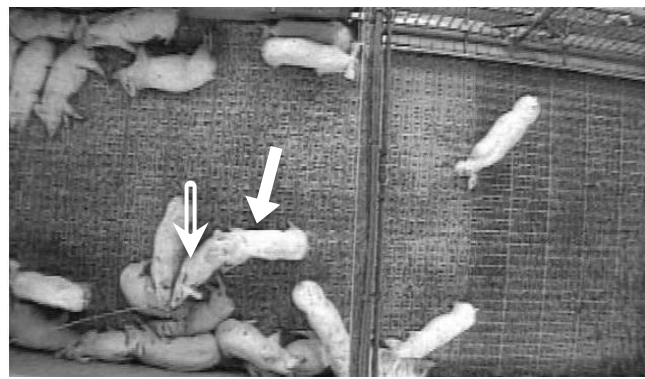

(f3)

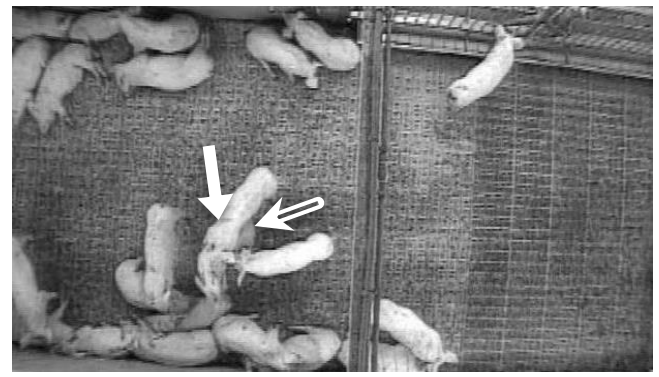

(f5)

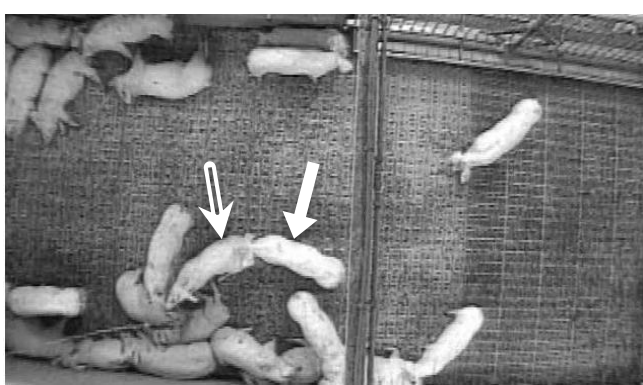

(f2)

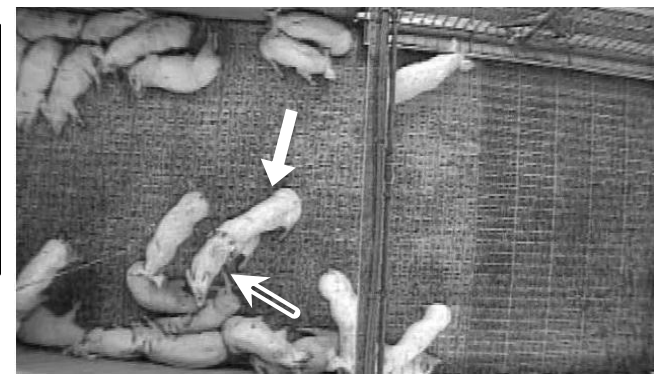

(f4)

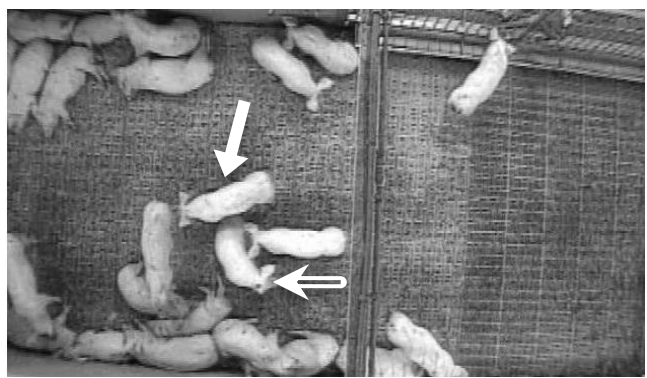

(f6)

Fig.2. Mounting behaviour in pig. (f1- f2) getting close, (f3-f5) mounting happened, (f6) getting away/ mounting finished.

141 In order to find the distance between two pigs in a mounting event, it was necessary to identify the head, tail and two sides of pigs. As a tool, analysis of the body contour of a pig was suggested by Kashiha et al. (2013), but in this study the long distance from the lens (camera) to the object (pig), low quality of images and the background noise made the method inaccurate. 
(a)
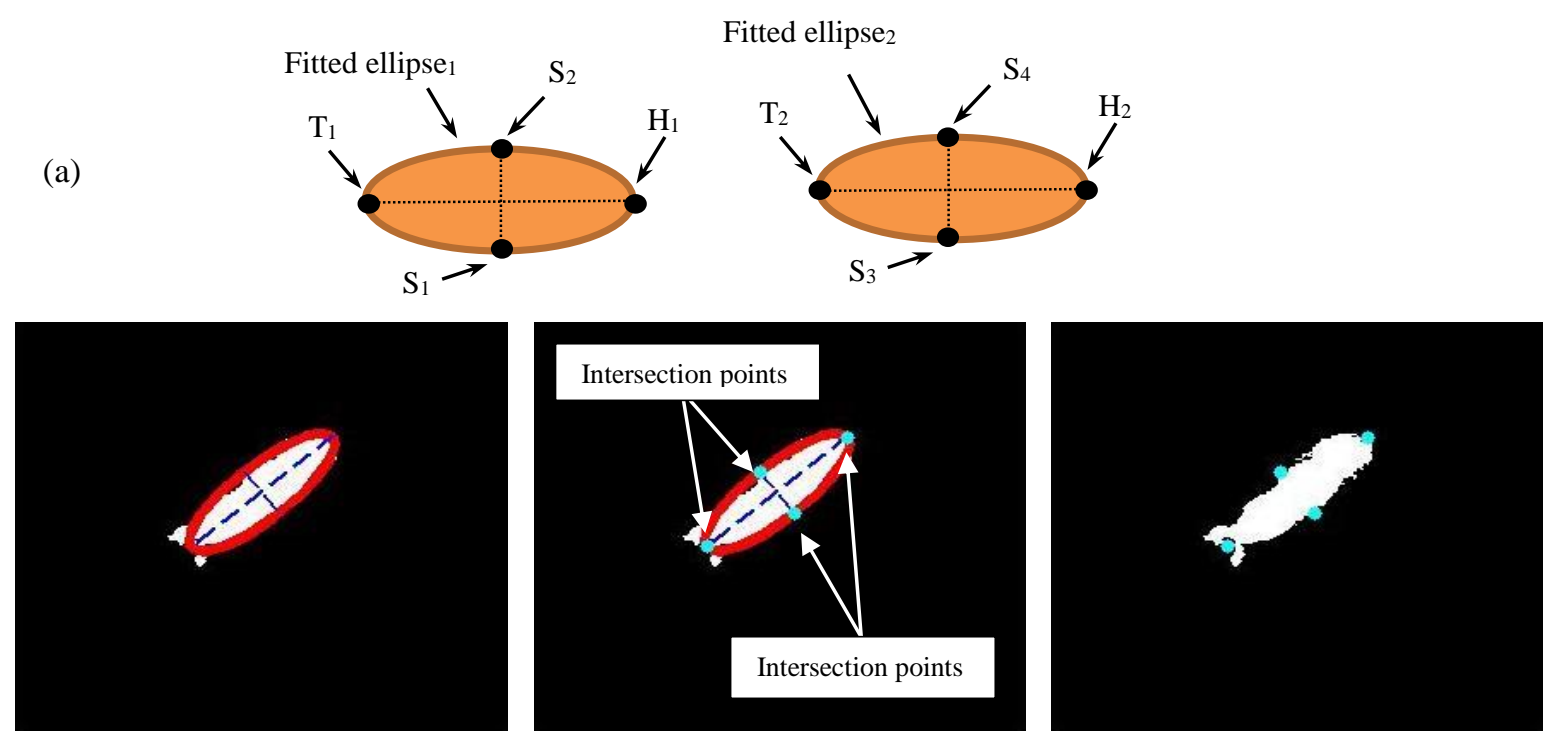

(b)

150

Fig.3. Intersection points of major and minor axis and ellipse for finding the position of head, tail and sides in pigs. (a); T,H and $\mathrm{S}$ in two fitted ellipses, (b); the T, $\mathrm{H}$ and $\mathrm{S}$ in a pig in binary image.

Therefore, in this work, the intersections of the major and minor axis with the ellipse have

154 been considered as tail/head and sides respectively (Fig. 3), named as T, H, S and then the 155 Euclidean distance $(\mathrm{Ed}) \quad\left(E d\left(H_{i}, T_{j}\right)\right)=\sqrt{ }$
$156 \sqrt{\sum_{i=1}^{n}\left(H_{i}-S_{i}\right)^{2}}$ of each pair calculated as follows:

157 Matrix of head and/or tail for n pigs (T, H): $\left[\begin{array}{cc}T_{1} & H_{1} \\ T_{2} & H_{2} \\ \cdot & \cdot \\ \cdot & \cdot \\ \cdot & \cdot \\ \cdot & \cdot \\ \cdot & \cdot \\ \cdot & \cdot \\ T_{n-1} & H_{n-1} \\ T_{n} & H_{n}\end{array}\right]$

158 Matrix of pig sides for n pigs $(\mathrm{S}, \mathrm{S}):\left[\begin{array}{cc}S_{1} & S_{2} \\ S_{3} & S_{4} \\ \cdot & \cdot \\ \cdot & \cdot \\ \cdot & \cdot \\ \cdot & \cdot \\ \cdot & \cdot \\ \cdot & \cdot \\ S_{2 n-3} & S_{2 n-2} \\ S_{2 n-1} & S_{2 n}\end{array}\right]$ 
$159 \stackrel{(E q .1)}{\longrightarrow} E d\left(T_{1},\left[\begin{array}{c}H_{2} \\ H_{3} \\ \cdot \\ \cdot \\ H_{n-1} \\ H_{n}\end{array}\right]\right), E d\left(T_{2},\left[\begin{array}{c}H_{1} \\ H_{3} \\ \cdot \\ \cdot \\ H_{n-1} \\ H_{n}\end{array}\right]\right) \ldots E d\left(T_{n},\left[\begin{array}{c}H_{1} \\ H_{2} \\ \cdot \\ \cdot \\ H_{n-2} \\ H_{n-1}\end{array}\right]\right)$

$\stackrel{(E q .1)}{\longrightarrow} \operatorname{Ed}\left(T_{1},\left[\begin{array}{c}T_{2} \\ T_{3} \\ \cdot \\ \cdot \\ T_{n-1} \\ T_{n}\end{array}\right]\right), E d\left(T_{2},\left[\begin{array}{c}T_{1} \\ T_{3} \\ \cdot \\ \cdot \\ T_{n-1} \\ T_{n}\end{array}\right]\right) \ldots E d\left(T_{n},\left[\begin{array}{c}T_{1} \\ T_{2} \\ \cdot \\ \cdot \\ T_{n-2} \\ T_{n-1}\end{array}\right]\right)$

$161 \stackrel{(E q .1)}{\longrightarrow} E d\left(H_{1},\left[\begin{array}{c}H_{2} \\ H_{3} \\ \cdot \\ \cdot \\ H_{n-1} \\ H_{n}\end{array}\right]\right), E d\left(H_{2},\left[\begin{array}{c}H_{1} \\ H_{3} \\ \cdot \\ \cdot \\ H_{n-1} \\ H_{n}\end{array}\right]\right) \ldots E d\left(H_{n},\left[\begin{array}{c}H_{1} \\ H_{2} \\ \cdot \\ \cdot \\ H_{n-2} \\ H_{n-1}\end{array}\right]\right)$

$162 \stackrel{\text { (Eq.1 and } 2)}{\longrightarrow} E d\left(T_{1},\left[\begin{array}{c}S_{3} \\ S_{4} \\ \cdot \\ \cdot \\ S_{2 n-1} \\ S_{2 n}\end{array}\right]\right), E d\left(T_{2},\left[\begin{array}{c}S_{1} \\ S_{2} \\ \cdot \\ \cdot \\ S_{2 n-1} \\ S_{2 n}\end{array}\right]\right) \ldots . E d\left(T_{n},\left[\begin{array}{c}S_{1} \\ S_{2} \\ \cdot \\ \cdot \\ S_{2 n-3} \\ S_{2 n-2}\end{array}\right]\right)$

$163 \stackrel{(E q .1 \text { and } 2)}{\longrightarrow} E d\left(H_{1},\left[\begin{array}{c}S_{3} \\ S_{4} \\ \cdot \\ \cdot \\ S_{2 n-1} \\ S_{2 n}\end{array}\right]\right), E d\left(H_{2},\left[\begin{array}{c}S_{1} \\ S_{2} \\ \cdot \\ \cdot \\ S_{2 n-1} \\ S_{2 n}\end{array}\right]\right) \ldots . E d\left(H_{n},\left[\begin{array}{c}S_{1} \\ S_{2} \\ \cdot \\ \cdot \\ S_{2 n-3} \\ S_{2 n-2}\end{array}\right]\right)$

165 Based on the typical behaviour of pigs, they normally move forward and mount with their 166 front legs onto a part of the mounted pig's body. As a result, in a sequence of frames, the 167 distance from the head of one pig to the other pig (head or tail) could be obtained from its direction of movement, as well as the distances between head of one pig to both sides of other 169 pigs. By finding the region of interest (ROI) for each participant pair (two pigs) with an Ed 170 (Eq. 1) less than a defined value (here, about half of the major axis length), the possibility of mounting events has been investigated in the algorithm, and the $x-y$ coordinates of the centre of the two pigs in the ROI recorded for the next steps. Note that as the mounting event is performed, the Ed between the head of first pig and the tail/head or side of the second one has been reduced from the previous frame and the two pigs considered as one in the algorithm; 
here the length of two pigs (length of major axis in fitted ellipse) will be changed to approximately 1.3 to 2 pig lengths if the pig is mounting from behind the second one, and the length of major and minor axis will be around 1.3-1.8 pig lengths if the pig is mounting from the side of another pig. So, if the length of the ellipse(s) was between the aforementioned value and the $x-y$ coordinates of the ellipse located in the ROI, the mounting behaviour was declared. Furthermore, if two pigs were standing close to each other without any mounting event, the algorithm just fitted an ellipse to each of the pigs and no mounting behaviour was specified.

\section{Results and discussion}

Fig. 4 shows the Ed between two points (H/T, H/S of one pig to another one); it could be inferred that the distances between the mounting and mounted pig declined before the mounting event happened. The algorithm only detected an Ed less than 43 (in pixels) (Fig. 5) as the ROI in this study. Fig. 5 illustrates the changes in Ed before and after the ROI for a mounting behaviour has been identified; when the $\mathrm{Ed}=0$ the mounting events happened (during time 5-14 s, $17 \mathrm{~s}, 27-33 \mathrm{~s}$ and $35 \mathrm{~s}$ ) and it can be seen that there was a discontinuous mounting event. The major axis length of the fitted ellipse for both mounting and mounted pigs for a mounting event which happened from the back is shown in Fig. 6. According to the diagram, the length of each pig was around 80 (pixels) (see Table 1) and, as the mounting event happened at second 5, the algorithm considered the mounting and mounted pigs as one pig and fitted an ellipse with a bigger major length. At the beginning of the mounting event, the length of the major axis was larger and it then declined over time as the mounting pig demonstrated pelvic thrusts (Hintze et al., 2013). Fig. 7 illustrates the major and minor axis length of mounting and mounted pigs when the mounting event occurred from the side. Here, 
199 the major length during the mounting event was around 1.4 pig lengths, while the major axis

200 length in the mounting event was approximately 2 times one pig's minor length.

201

202

203

204

205

206

207

208

209

210

211

212

213

214

215

216

217

218

219

220

221

222

223

224

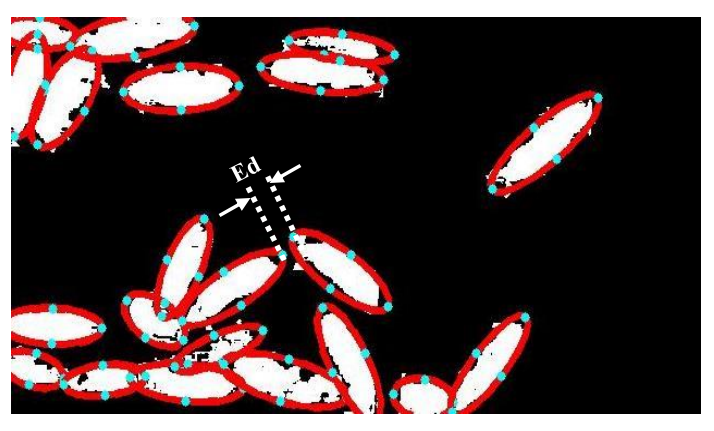

I

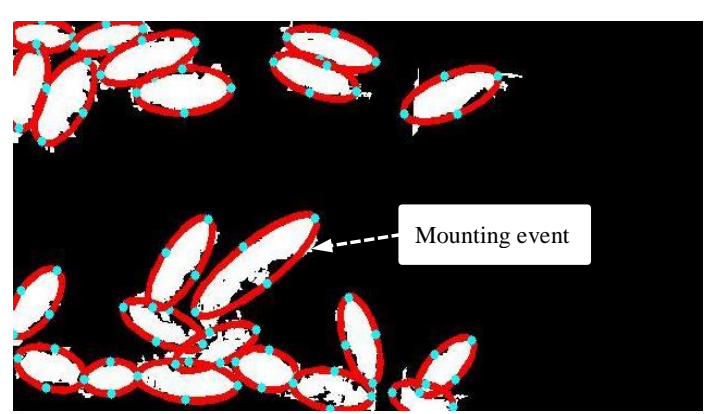

III
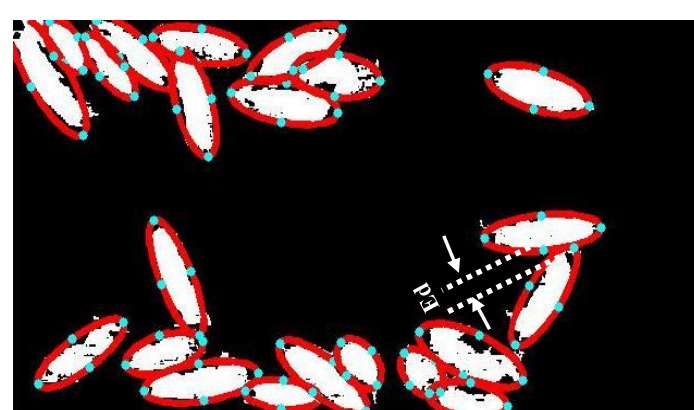

IV

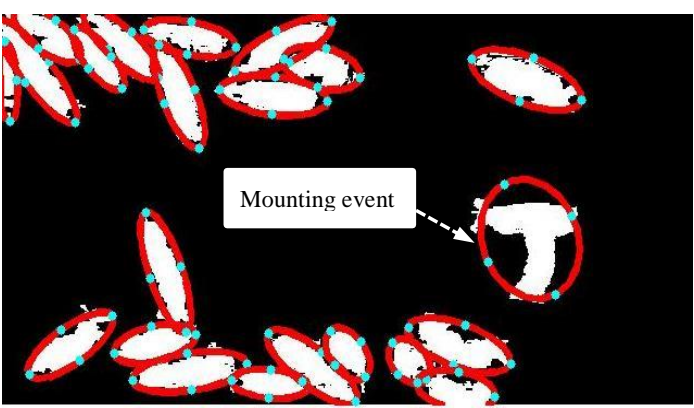

VI

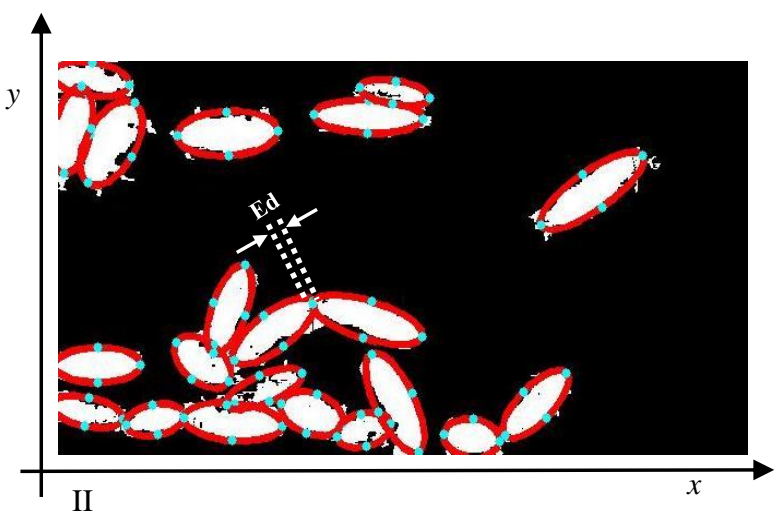

Euclidian distance between two points ( $\mathrm{H}$ and $\mathrm{T})$

$$
E d(H, T)=\sqrt{\left(H_{x}-T_{x}\right)^{2}+\left(H_{y}-T_{y}\right)^{2}}
$$

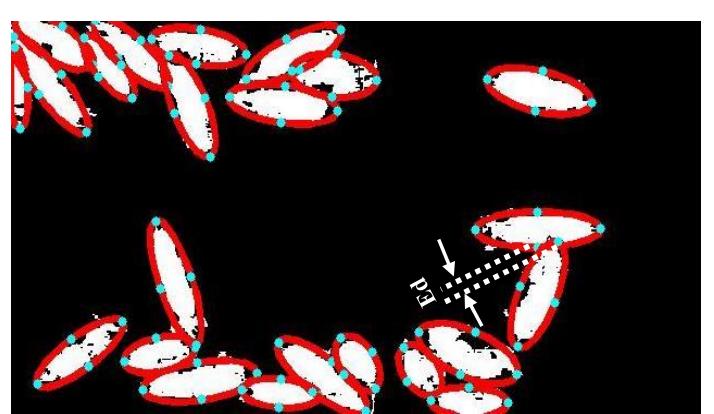

V

Euclidian distance between two points $(\mathrm{H}$ and $\mathrm{S})$ $E d(H, S)=\sqrt{\left(H_{x}-S_{x}\right)^{2}+\left(H_{y}-S_{y}\right)^{2}}$

Fig.4. The Ed between Tail and Head of two pigs during a mounting event. For a mount from behind: (I and II) the Ed declined, (III) mounting happened from the back giving a bigger ellipse. For a mount from the Side: (IV and V) the Ed declined, (VI) mounting happened from the side giving a bigger ellipse. 


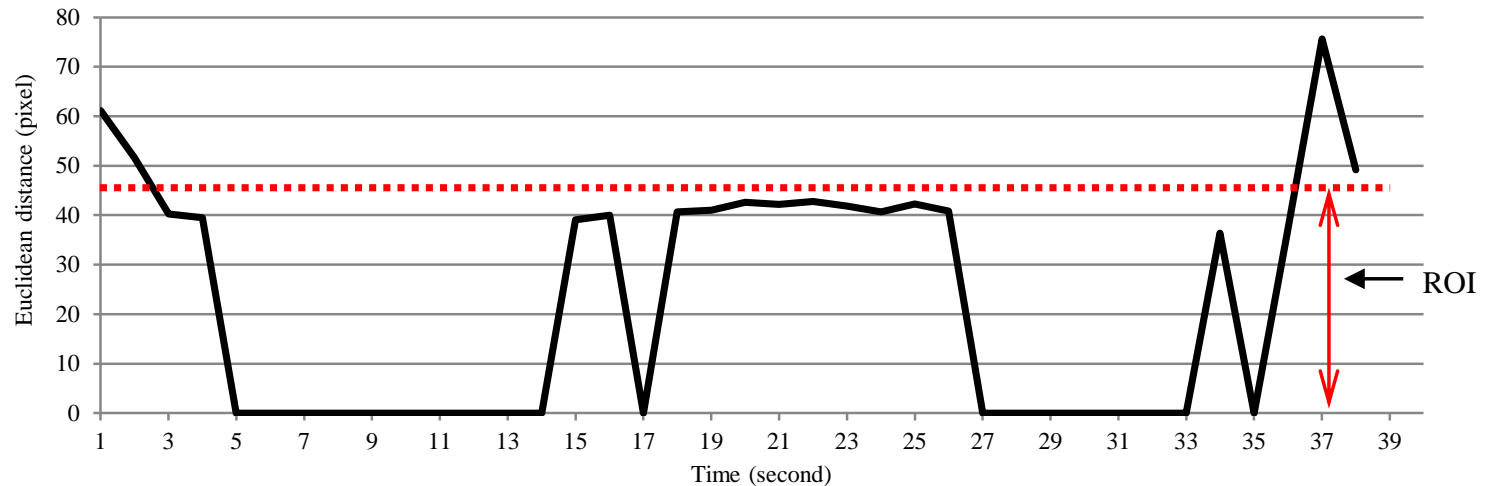

Fig.5. Euclidian distance between two pigs (mounting and mounted) and the ROI.

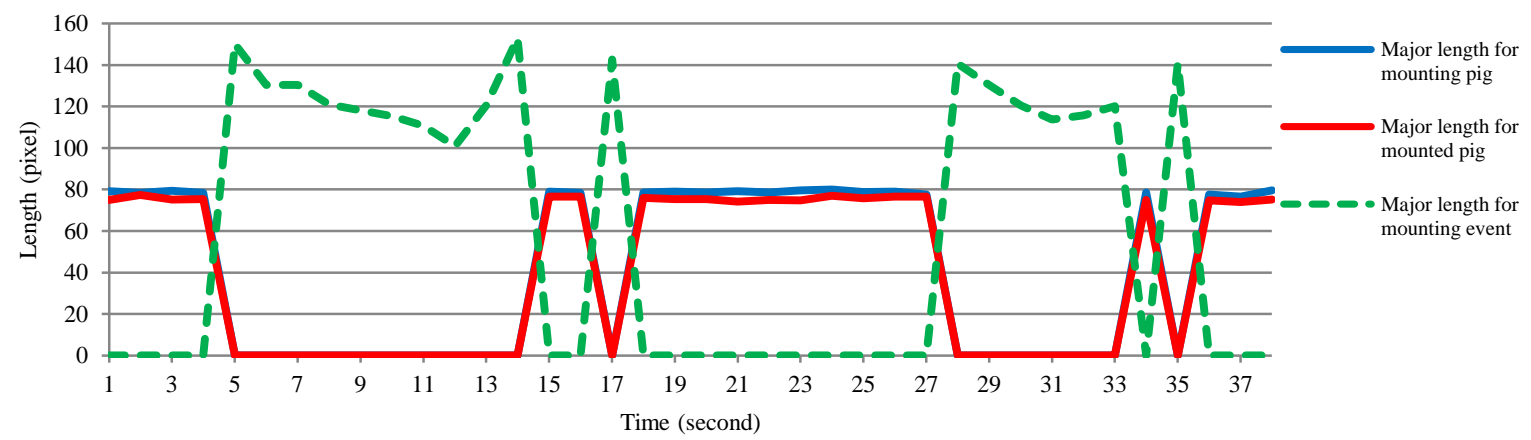

Fig.6. The major axis length of mounting and mounted pigs, along with the mounting event length, for a mounting event from the

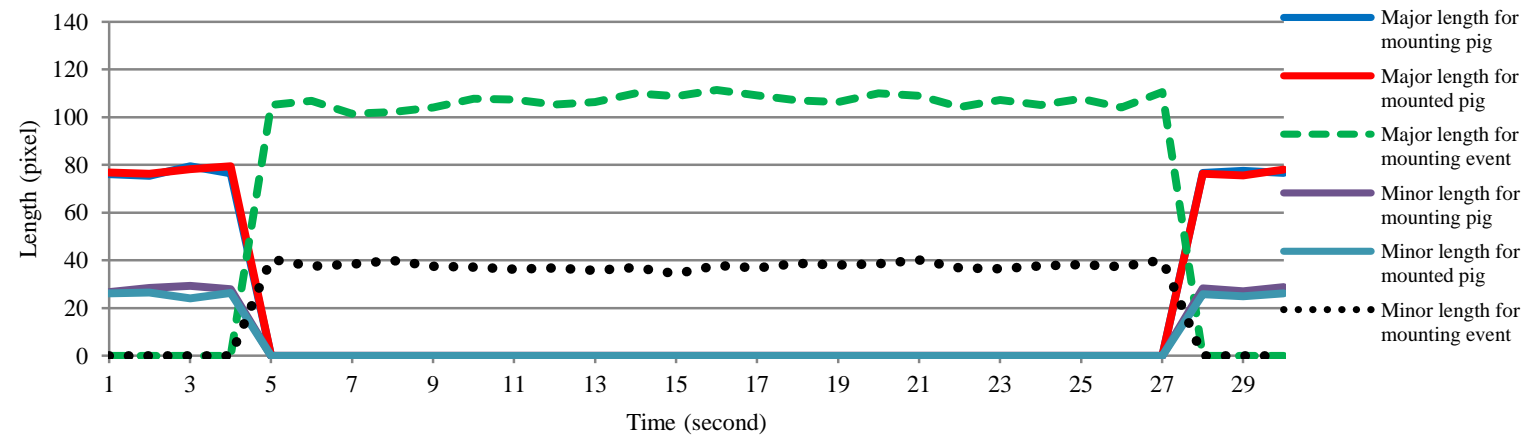

Fig.7. The major and minor axis length of mounting and mounted pigs along with mounting event length, for a mounting event from the side.

Table 1. Mean and standard deviation (SD) of major and minor axis length of pigs in ROI before and after of the mounting event.

\begin{tabular}{lccccccc}
\hline Time (second) & 1 & 2 & 3 & 4 & 27 & 28 & 29 \\
\hline Major axis length (pixel) \pm SD & $76.4 \pm 0.5$ & $75.8 \pm 0.6$ & $77.8 \pm 0.4$ & $76.8 \pm 0.6$ & $76.4 \pm 0.2$ & $76.9 \pm 0.6$ & $77.3 \pm 0.9$ \\
& & & & & & & \\
Minor axis length (pixel) \pm SD & $26.4 \pm 0.3$ & $27.4 \pm 0.8$ & $27.3 \pm 1.1$ & $26.7 \pm 0.6$ & $26.5 \pm 0.9$ & $25.9 \pm 1.2$ & $27.1 \pm 0.9$ \\
\hline
\end{tabular}


240 From the $200 \mathrm{~h}$ of recorded videos, a total of 120 mounting events were visually obtained. In

241 general, $1800 \mathrm{~s}$ of mounting events and 7,200 frames (4 frames per second) were obtained

242 from both pens during the study. The mounting events were manually validated from the

243 recorded video frames by an expert. The validation scales used for finding the performance of

244 the detection system were defined as in Table 2 (Firk et al., 2002; Pourreza et al., 2012; Tsai 245 and Huang, 2014).

246

Table 2. Definition of validation parameters

\begin{tabular}{llc}
\hline Scale & Definition & Value \\
\hline True positive (TP) & Mounting event considered as mounting event & 4753 \\
False positive (FP) & Non-mounting event considered as mounting event & 247 \\
True negative (TN) & Non-mounting event considered as non-mounting event & 1925 \\
False negative (FN) & Mounting event considered as non-mounting event & 275 \\
\end{tabular}

Sensitivity $=\frac{T P}{T P+F N} \times 100 \longrightarrow \frac{4753}{4753+275}=94.5 \%$

Specificity $=\frac{T N}{T N+F P} \times 100 \longrightarrow \frac{1925}{1925+247}=88.6 \%$

Accuracy $=\frac{T P+T N}{T P+F P+T N+F N} \times 100 \longrightarrow \frac{4753+1925}{4753+247+1925+275}=92.7 \%$

250 The result obtained from the validation of the algorithm shows a good mounting detection 251 rate with satisfactory sensitivity (94.5\%), specificity (88.6\%) and accuracy (92.7\%). 252 According to the criteria of Table 2, some mounting frames were not recognized and there were some false positives. These errors sometimes occurred because the project was carried out in a commercial farm where there was a water pipe in the middle of each pen $(2.5 \mathrm{~m}$ from 
the floor) and some mounting events happened in this invisible area. Furthermore, when the apparent mounting event happened near a pen wall and/or when the mounting pig contacted or tried to contact a pig from a neighbouring pen, drank from the attached nipple drinker or licked the wall (Hintze et al., 2013), and due to the low image quality, the system could not properly distinguish the wall and pigs.

It is clear that the mounting behaviours in pigs need different detection methods from those of some other species due to differences in the nature of their behaviours. For example, the mounting behaviour in cows contains a few seconds of following behaviours (Tsai and Huang, 2014), in which the mounting cow closely follows the mounted cow, and then a jumping or mounting event happens. Tsai and Huang, (2014) have shown that, because of following behaviours in cows, using the motion analysis of mounting events could be a good technique for mounting detection. In contrast, mounting in the pig often happens without any preceding following. Furthermore, the mounted pig may be sitting down or moving away during the event, so using the recommended method for cows may not be applicable in pig behaviour detection.

This study has shown that binary image and fitted ellipse features can be used to extract features related to mounting behaviour among pigs. However, the system could not identify all mounting events, because the CCTV camera could not always detect the pig's body and make a clear distinction between pigs and wall or pigs and background (pen). This problem might be overcome by using 3D image data (i.e. time-to-flight, Microsoft Kinect sensor) which has the advantages of elimination errors related to animal colours, background and different ambient lighting (Kongsro, 2014), animal body detection in more detail (Weber et al., 2014) and pictures with higher resolution. However, using expensive cameras with better colour and object detection in commercial farms, in an environment with high levels of humidity, dust and ammonia, and their associated detrimental effects on electronics, may not 
be economically acceptable for farm managers. So possibilities for improving the algorithm for images from simple CCTV cameras or using other methods need to be considered in future research.

To date, no previous studies have been carried out to automatically detect pig mounting behaviours. The technique proposed here can automatically detect mounting events among pigs, even in commercial farm conditions. The method could be a valuable tool to aid farmers to increase animal welfare and health, and reduce injuries and economic losses, particularly as the use of entire males becomes more common. As the pigs grow larger, the mounted pigs may have increased risk of injury (Clark and D'Eath, 2013), and may be mounted more frequently by other pigs. So, with accurate information about the mounting events, the farmer can move quickly to address problem pens or seek interventions. Additionally, automated tracking of the time course and frequency of mounting behaviours within pens could facilitate the work of researchers exploring methods of prevention or alleviation of this behavioural problem.

\section{Conclusion}

In this study, automatic detection of mounting events among pigs, based on ellipse fitted features, was reported. A background subtraction method has been used for finding pigs in images and, after removing noise from binary images, $x-y$ coordinates of each binary image were used for localization of each pig in image (ellipse fitting technique). The Ed distances from head/tail of one pig to another and head/tail to sides of second pig were calculated for defining the ROI and, as the mounting event happened in the ROI, the size of two pigs combined (new fitted ellipse) altered to that of 1.3-2 pigs. The performance of the algorithm showed a high level of accuracy, so this method could contribute in the future as an important and economically feasible technique in commercial pig farms. This automatic method is an 
important step for developing an automatic system for making the farm management easier, cheaper and more efficient in use of manpower.

\section{Acknowledgments}

The authors wish to thank the Innovate UK project 101829 "Green Pigs" and Midland Pig Producers for access to commercial pig facilities.

\section{References}

Costa, A., Ismayilova, G., Borgonovo, F., Viazzi, S., Berckmans, D., Guarino, M., 2014. Image-processing technique to measure pig activity in response to climatic variation in a pig barn. Anim. Prod. Sci. 54, 1075-1083.

\section{Clark, C.C.A., D’Eath, R.B., 2013. Age over experience: Consistency of aggression and} mounting behaviour in male and female pigs. Appl. Anim. Behav.Sci. 147(1-2), 81-93.

Faucitano, L., 2001. Causes of skin damage to pig carcasses. Can. J. anim. Sci. 81(1), 39-45.

Firk, R., Stamer, E., Junge, W., Krieter, J., 2002. Automation of oestrus detection in dairy cows: a review. Livest. Prod. Sci. 75(3), 219-232.

\footnotetext{
Gonzalez, R., Woods, R., 2007. Digital image processing, $3^{\text {rd }}$ ed. Prentice-Hall.
} 
Harley, S., Boyle, L.A., O'Connell, N.E., More, S.J., Teixeira, D.L., Hanlon, A., 2014. Docking the value of pigmeat? Prevalence and financial implications of welfare lesions in Irish slaughter pigs. Anim. Welfare. 23, 275-285.

Hemsworth, P.H., Tilbrook, A.J., 2007. Sexual behavior of male pigs. Horm. Behav. 52(1), $39-44$.

Hintze, S., Scott, D., Turner, S., Meddle, S.L., D’Eath, R.B., 2013. Mounting behaviour in finishing pigs: Stable individual differences are not due to dominance or stage of sexual development. Appl. Anim. Behav. Sci. 147(1-2), 69-80.

Geys, I., Gool, L.V., 2007. View synthesis by the parallel use of GPU and CPU. Image. Vision. Comput. 25(7), 1154-1164.

Gronskyte, R., Clemmensen, L.H., Hviid, M.S., Kulahci, M., 2015. Pig herd monitoring and undesirable tripping and stepping prevention. Comput. Electron. Agric. 119, 51-60.

Kashiha, M., Bahr, C., Haredasht, S.A., Ott, S., Moons, C.P.H., Niewold, T.A., Ödberg, F.O., Berckmans, D., 2013. The automatic monitoring of pigs water use by cameras. Comput. Electron. Agric. 90, 164-169.

Kongsro, J., 2014. Estimation of pig weight using a Microsoft Kinect prototype imaging system. Comput. Electron. Agric. 109, 32-35. 
Lu, M., Xiong, Y., Li, K., Liu, L., Yan, L., Ding, Y., Lin, X., Yang, X., Shen, M., 2016. An automatic splitting method for the adhesive piglets' gray scale image based on the ellipse shape feature. Comput. Electron. Agric. 120, 53-62.

Mortensen, A.K., Lisouski, P., Ahrendt, P., 2016. Weight prediction of broiler chickens using 3D computer vision. Comput. Electron. Agric. 123, 319-326.

Nasirahmadi, A., Richter, U., Hensel, O., Edwards, S., Sturm, B., 2015. Using machine vision for investigation of changes in pig group lying patterns. Comput. Electron. Agric. 119, 184-190.

O’Leary, P., 2004. Direct and specific least-square fitting of hyperbolæ and ellipses. J. Electron. Imaging. 13(3), 492-503.

Otsu, N., 1979. A threshold selection method from gray-level histograms. IEEE Trans. Syst. Man Cybern. 9(1), 62-66.

Pourreza, A., Pourreza, H., Abbaspour-Fard, M., Sadrnia, H., 2012. Identification of nine Iranian wheat seed varieties by textural analysis with image processing. Comput. Electron. Agric. 83,102-108.

Prunier, A., Bonneau, M., von Borell, E.H., Cinotti, S., Gunn, M., Fredriksen, B., Giersing, M., Morton, D.B., Tuyttens, F.A.M., Velarde, A., 2006. A review of the welfare consequences of surgical castration in piglets and the evaluation of non-surgical methods. Anim. Welfare. 15(3), 277-289. 
378 Rydhmer, L., Zamaratskaia, G., Andersson, H. K., Algers, B., Lundström, K., 2004. Problems with aggressive and sexual behaviour when rearing entire male pigs. In Proc. 55th Ann. Meet. Europ. Assoc. Anim. Prod., Bled.

Rydhmer, L., Zamaratskaia, G., Andersson, H.K., Algers, B., Guillemet, R., Lundström, K., 2006. Aggressive and sexual behaviour of growing and finishing pigs reared in groups, without castration. Acta Agric. Scand., A. 56(2), 109-119. comfort for group-housed pigs. Comput. Electron. Agric. 62(1), 15-21.

Thomsen, R., Bonde, M., Kongsted, A.G., Rousing, T., 2012. Welfare of entire males and females in organic pig production when reared in single-sex groups. Livest. Sci.

399

Shao, B., Xin, H., 2008. A real-time computer vision assessment and control of thermal

Stavrakakis, S., Li, W., Guy, J.H., Morgan, G., Ushaw, G., Johnson, G.R., Edwards, S.A., 2015. Validity of the Microsoft Kinect sensor for assessment of normal walking patterns in pigs. Comput. Electron. Agric. 117, 1-7.

Teixeira, D.L., Boyle, L.A., 2014. A comparison of the impact of behaviours performed by entire male and female pigs prior to slaughter on skin lesion scores of the carcass. Livest. Sci. 170, 142-149.

Tsai, D., Huang, C., 2014. A motion and image analysis method for automatic detection of estrus and mating behavior in cattle. Comput. Electron. Agric. 104, 25-31. 
403 Viazzi, S., Ismayilova, G., Oczak, M., Sonoda, L.T., Fels, M., Guarino, M., Vranken, E., 404 Hartung, J., Bahr, C., Berckmans, D., 2014. Image feature extraction for classification 405 of aggressive interactions among pigs. Comput. Electron. Agric. 104, 57-62.

406

407 Wang, Z., Wu, W., Xu, X., Xue, D., 2007. Recognition and location of the internal corners 408 of planar checkerboard calibration pattern image. Appl. Math. Comput. 185(2), 894409 906.

410 Weber, A., Salau, J., Haas, J.H., Junge, W., Bauer, U., Harms, J., Suhr, O., Schönrock, K., 411 Rothfuß, H., Bieletzki, S., Thaller, G., 2014. Estimation of backfat thickness using extracted traits from an automatic 3D optical system in lactating Holstein-Friesian cows. Livest. Sci. 165, 129-137. 